\title{
DIE SPRACHLICHE NORM UND IHRE (IN)VARIANTEN ALS FESTE GRUNDLAGEN FÜR DIE EVALUATION VON SPRACHLICHEN AUSDRÜCKEN
}

Der Begriff „sprachliche Norm“ ist von entscheidender Bedeutung für die Problematik des sprachlichen Fehlers. Man braucht die Norm als eine Beurteilungsinstanz, um feststellen zu können, ob irgendwelche sprachliche Struktur das Merkmal „fehlerhaft“ oder „korrekt" aufweist. An der Stelle muss man bemerken, dass die Realisierung der Norm nicht immer die Wahl zwischen „korrekt" und „falsch“ bedeutet. Denn in manchen Fällen ist es unmöglich, eine scharfe Grenze zwischen „richtig“ und „falsch“ zu ziehen. Wenn eine Norm realisiert werden soll, so stehen einem manchmal nicht nur eine bestimmte Form, sondern mehrere Formen zur Wahl. Bei manchen Ausdrücken ist es unmöglich, die einzig korrekte Form zu wählen. Dagegen in vielen Fällen ist es unproblematisch, diejenigen sprachlichen Formen zu zeigen, die eindeutig gegen die Norm verstoßen. Die gelegentliche Unmöglichkeit, das Richtige vom Falschen zu trennen, scheint das größte Problem im Bereich der Norm zu sein. Juhász führt dazu ein zutreffendes Zitat von Dauzat: „Nichts ist weniger wissenschaftlich als die Absicht, alles in zwei Teile teilen zu wollen, säuberlich das Richtige vom Falschen trennen zu wollen und zu behaupten, dies dürfe man sagen und jenes nicht. Die Fehler müssen demgemäß abgestuft werden“ (Juhász 1970, S. 47f.). Da die vorliegenden Ausführungen einen synchronischen Charakter haben, muss man sich dessen bewusst sein, dass es Schwierigkeiten bereiten kann, im Fall von „Norm“ von Synchronie zu sprechen. Der Grund dafür ist die permanente Veränderung der Sprache, die im Grunde genommen ein Prozess ist, der unaufhaltsam verläuft und im Wesen der Sprache verankert ist. Auch der individuelle Faktor, d.h. ein jeder Sprachbenutzer, soll nicht außer Acht gelassen werden. Juhász ist der Ansicht, dass es „schon von der Funktion der Sprache her nicht möglich ist, den Gebrauch sämtlicher Elemente als Invarianten zu betrachten, sondern daß die Stellungnahme des Sprachausübenden zur Wirklichkeit in zahlreichen Fällen für die Wahl des sprachlichen

* Dr. Tomasz Maras, Universität Łódź, Philologische Fakultät, Lehrstuhl für Deutsche und Angewandte Sprachwissenschaft, 90-236 Łódź, Pomorska 171/173. 
Elements entscheidend ist" (Juhász 1970, S. 37). Dies erschwert die Normensetzung und wirft die Frage nach ihrem Sinn auf. Die Dynamik der Veränderungen ist nicht so schnell, dass die Normensetzung unmöglich wird. In dem vorliegenden Artikel wird der Standpunkt vertreten, dass die Norm vor allem dazu verhilft, die Sprache zu systematisieren und zu pflegen (allerdings nicht im puristischen Sinne). Bei der Bestimmung der Korrektheit bzw. Fehlerhaftigkeit eines gegebenen Ausdrucks z.B. in der Interferenz-Untersuchung sollen die zuverlässigsten Regelwerke der deutschen Sprache zu Hilfe genommen werden. Wenn sie keine eindeutigen Antworten geben, kann mit Hilfe von Sprachgefühl einiger Muttersprachler entschieden werden, ob eine konkrete Form (stilistisch) korrekt ist oder nicht. Das wichtigste Kriterium dabei ist, wie sich die Form auf die sprachliche Kommunikation auswirkt.

Im Folgenden werden verschiedene Auffassungen der sprachlichen Norm gezeigt, um das Phänomen in seiner Komplexität zu erfassen. Die sprachliche Norm wird in der einschlägigen Literatur definiert als „Gesamtheit der in einer Sprachgemeinschaft (in Bezug auf Rechtschreibung, Aussprache, Grammatik u. Stil) als üblich und richtig festgelegten Regeln" (Duden Deutsches Universalwörterbuch, S. 1493). Diese Normauffassung entstammt dem Duden-Universalwörterbuch und spricht 3 wichtige Aspekte der Norm an: die Sprachgemeinschaft (in der die Norm gilt), die Regeln (die für die Normierung unentbehrlich sind) und Geltungsbereiche der Norm (Rechtschreibung, Aussprache, Grammatik, Stil). Semenjuk betont die Stabilisierung und den Traditionsbezug der Normelemente, die durch die gesellschaftliche Sprachausübung gewählt und fixiert wurden, indem er die Norm folgendermaßen versteht: „Gesamtheit der stabilsten, traditionellen, durch die gesellschaftliche Sprachpraxis ausgewählten und fixierten Realisierungen der Elemente der Sprachstruktur" (Semenjuk 1975, S. 459). Für ihn spielt die Tradition bei der Norm eine wichtige Rolle. Die Tradition ist mit der diachronischen Auffassung der Norm verbunden, die in den vorliegenden Ausführungen nicht näher thematisiert wird.

Nerius greift in seiner Normauffassung u.a. die Frage der Invarianten auf. Er interpretiert die Norm nicht als die Gesamtheit der in einer gegebenen Gesellschaft gebrauchten Sprachformen und schreibt:

Die Norm umfaßt in der Regel nicht die Gesamtheit der in einer Sprachgemeinschaft gebräuchlichen sprachlichen Erscheinungen. Neben dem invarianten Bereich gibt es eine jeweils mehr oder weniger große Zahl von Varianten. In dem Verhältnis von invariantem Bereich und Varianten liegt das Problem der sprachlichen Norm... Das Verhältnis des invarianten Bereichs zu den Varianten ist in den einzelnen Teilen der Sprache unterschiedlich, d.h. die Norm ist nicht überall gleichermaßen deutlich ausgeprägt. Relativ am stärksten gefestigt erscheint sie... im grammatischen... Bereich, während etwa in der Aussprache eine außerordentlich große Variationsbreite besteht. Ein Versuch, das Sprachganze in Hinsicht auf die Norm allgemein zu differenzieren, wird auf der Grundlage statistischer Ermittlungen zu unterscheiden haben:

- den invarianten Bereich, 
- den Bereich der fakultativen, mehr oder weniger gleichberechtigten bzw. gleich häufigen Varianten,

- einen Bereich solcher Varianten, die aufgrund ihrer begrenzten Anwendung oder ihres im Verhältnis zu den jeweiligen parallelen Formen relativ geringen Vorkommens von der Mehrzahl der Sprecher oder Schreiber als fehlerhaft, als unregelmäßig oder doch immerhin als ungewöhnlich empfunden werden (Nerius 1967, S. 11).

Nerius' wichtigster Ansatz ist hier die Einteilung in den invarianten Bereich und in Varianten. In der Relation zwischen dem invarianten Bereich und den Varianten besteht seiner Meinung nach das größte Problem bei der Bestimmung der sprachlichen Norm. Die Relation sei unterschiedlich in verschiedenen Teilen der Sprache. Am stärksten befestigt sieht Nerius die Norm in der Grammatik, wobei er anmerkt, dass auch hier (z.B. in der Aussprache) eine große Zahl von Variationen zu erwarten ist.

Althaus/Henne/Wiegand nehmen die Komplexität der Norm ins Visier, indem sie auf die Existenz von Sender (A), Rezipienten (B), Kontext (C) und Zweck (D) sowie „Normsetzer“ und „Normüberwacher“" hinweisen:

Der Terminus ,Sprachnorm' ist folglich ein mehrstelliger Prädikator mindestens dieser Komplexität: eine Sollensforderung ist nach dem Willen von A für einen Personenkreis B unter den Situationsbedingungen C in Bezug auf den Zweck D eine Norm. Damit ist berücksichtigt, daß es Normensetzer (und Normenüberwacher) gibt, daß Normen oft spezifische Adressaten haben, daß sie in ihrem Geltungsbereich bzw. auf Sprechsituationen und/oder kommunikative Absichten beschränkt sind und daß ihre Konzeption und Durchsetzung interessengebunden ist (was sich allerdings nur für eine Teilmenge rekonstruieren läßt) (Althaus, Henne, Wiegand 1980, S. 364).

Nach ihrer Auffassung erfüllt die Norm auch kommunikative Aufgaben:

In diesem soziolinguistischen Normbegriff (,Kommunikationsnormen“) wird somit den sozialen Merkmalen der Sprachnormen und den Handlungsaspekten ihres Wirkens Rechnung getragen. Das Hauptmerkmal dieser Normen ist, daß sie sich auf eine kommunikative Adäquatheit des Sprechens beziehen. (...) (Althaus, Henne, Wiegand 1980, S. 364).

Für Skudrzykowa/Urban beginnt die Normauffassung schon auf der Ebene der Morpheme und umfasst die Regeln ihrer Verbindung und Aussprache bzw. schriftlicher Fixierung:

NORMA JĘZYKOWA - to zbiór wszystkich morfemów (najmniejszych cząstek wyrazowych, mających znaczenie w danym języku) oraz sposobów ich łączenia, wymawiania i zapisywania, które są w danym czasie uznane przez społeczeństwo za poprawne (Skudrzykowa, Urban 2000, S. 105).

Die Norm betrifft für sie alle Aspekte der sprachlichen Kommunikation und wird eingeteilt in Flexionsnorm, phonetische Norm, kommunikative Norm, lexikalisch-phraseologische Norm, orthographische Norm, syntaktische Norm, Wortbildungsnorm, stilistische und Aussprachenorm: 
Norma dotyczy wszystkich aspektów komunikowania się przy pomocy języka, mówimy więc o normie fleksyjnej, fonetycznej, komunikacyjnej, leksykalno-frazeologicznej, ortograficznej, składniowej, słowotwórczej, stylistycznej, wymawianiowej (Skudrzykowa, Urban 2000, S. 105).

Unter dem Aspekt der Kommunikationssituation unterscheiden Skudrzykowa/ Urban zwischen Gebrauchsnorm [,norma użytkowa (potoczna)"] und hochsprachlicher Norm (norma wzorcowa):

Ze względu na sytuację komunikowania, stopień jej oficjalności, norma jest wewnętrznie zróżnicowana i dlatego wyróżnić możemy normę użytkową (potoczną) oraz staranną, wzorcową (Skudrzykowa, Urban 2000, S. 105).

Der erstere Normtyp - die Gebrauchsnorm - wird im folgenden Kapitel erörtert.

\title{
1. Die Gebrauchsnorm
}

Die Gebrauchsnorm scheint insofern wichtig zu sein, als es bei vielen sprachlichen Ausdrücken, denen man bei verschiedenen Formen des Sprachgebrauchs (z.B. beim Übersetzen) begegnet, nicht möglich ist, die sprachliche Wirklichkeit in „korrekt“" und „falsch“ zu teilen. Die Termini „Gebrauchsnorm“ (poln. ,norma użytkowa“) sowie „Usus“ (poln. „uzus“) machen uns bewusst, dass sich sprachliche Mittel nicht innerhalb klarer und strenger Normen festlegen lassen. $\mathrm{Zu}$ Gebrauchsnorm äußert sich Markowski in folgenden Worten:

\begin{abstract}
Norma użytkowa obejmuje zestaw wyrazów, ich form i połączeń charakterystycznych dla tych typów kontaktów, w których język traktuje się jako wartość użytkową, a konkretnie jako narzędzie (przekazu, informacji, perswazji itd.). Chodzi więc o zespół środków językowych używanych w kontaktach swobodnych, przede wszystkim nieoficjalnych, o różnorodnej tematyce. Opisując pewne elementy językowe jako pochodzące z poziomu normy użytkowej, a więc aprobując je na tym poziomie, bierze się pod uwagę stopień rozpowszechnienia pewnego środka językowego, jego użyteczność, sprawność komunikatywną (np. ekonomiczność, prostotę, wyrazistość), w mniejszym zaś stopniu - zgodność z tradycją czy systemem językowym (Markowski 2005, S. 1627f.).
\end{abstract}

Aus seiner Definition wird ersichtlich, dass die Gebrauchsnorm sprachliche Formen und ihre Verbindungen erfasst, die für solche sprachlichen Kontakte charakteristisch sind, wo die Sprache als Werkzeug zur Information oder z.B. Persuasion gilt. Es geht hier vor allem um inoffizielle Kontakte, wo die sprachlichen Mittel weniger mit der sprachlichen Tradition und Sprachsystem vereinbar und nicht unbedingt korrekt sind. Trotzdem sind sie einfach, ökonomisch und deutlich und als solche erfüllen sie ohne Probleme ihre kommunikativen Funktionen.

Der Begriff „Gebrauchsnorm“ betrifft dieselbe Ebene wie der Terminus „Usus“, den Glück als eine Ebene mit sprachlichen Konventionen versteht, denen 
gemeinsam sei, dass sie schwächer als sprachliche Normen und nicht festgelegt (kodifiziert) sind:

Usus (lat. (übliche) Verwendung, Gebrauch) In der $\rightarrow$ Soziolinguistik und in Grammatikmodellen, die sprachl. Variation (z. B. die $\rightarrow$ Varietätengrammatik) zu erfassen suchen, Bez. für eine Ebene sprachl. Konventionen, die schwächer als $\rightarrow$ Regeln und $\rightarrow$ Sprachnormen und im Ggs. zu jenen weder explizit festgelegt noch den Sprechenden bewußt sind, jedoch als charakterist. und ,normal' für das Sprachverhalten eines durchschnittl. Mitglieds einer $\rightarrow$ Sprachgemeinschaft gelten (Glück 1993, S. 669).

Markowski weist mit seiner Usus-Definition auch darauf hin, dass die sprachlichen Elemente, die zum Usus gehören, die Grundlage für die Veränderungen innerhalb der sprachlichen Norm sind. Auf diese Weise können diejenigen Elemente, die immer populärer in einer Sprachgemeinschaft werden, die Usus-Ebene verlassen und ihren Platz in der kodifizierten Sprachnorm finden:

UZUS JĘZYKOWY jest to panujący w pewnym środowisku powszechny zwyczaj używania takich, a nie innych form językowych. Na uzus składają się te elementy językowe, które występują bardzo często w tekstach określonego typu i określonego środowiska. Są to zarówno te wyrazy, ich formy i połączenia, które zyskały ogólną aprobatę i wchodzą do normy językowej, jak i te składniki tekstów, które nie zyskały dotąd powszechnej aprobaty i mogą razić niektórych członków danej społeczności, niemniej mają charakter obiegowy i są tradycyjnie używane. (...) Zasób elementów należących do uzusu jest podstawą przemian zachodzących w normie językowej. Jeśli bowiem jakiś wyraz, forma wyrazowa, znaczenie leksykalne, połączenie składniowe czy frazeologiczne uzyska znaczny stopień rozpowszechnienia, to z czasem zostanie najprawdopodobniej skodyfikowane w obrębie normy: najpierw wejdzie do normy użytkowej, a po pewnym czasie może zostać zaaprobowane także w normie wzorcowej (Markowski 2005, S. 1688).

Die Begriffe „Gebrauchsnorm” und „Usus” spielen eine wichtige Rolle auch bei der Untersuchung der (Interferenz) Fehler, wo es in vielen Fällen nicht möglich ist, eindeutig eine fehlerhafte bzw. korrekte Form zu bestimmen. Man muss immer darauf achten, dass die Norm mehrere Varianten haben und sich ändern kann. Es kann passieren, dass ein Ausdruck, der ein eindeutiger Interferenzfehler war, dermaßen verbreitet wird, dass er letztendlich zur Ebene der kodifizierten Norm gelangt.

\section{Zum Terminus „Sprachgefühl“6}

Wenn man die Korrektheit eines Ausdrucks feststellen will, kann man dabei eines der traditionellen Verfahren gebrauchen und zwar sein eigenes Sprachgefühl bzw. das Sprachgefühl eines anderen Sprachbenutzers zu Hilfe nehmen. Somit wird man (bzw. andere Personen) zum Informanten in Bezug auf die Richtigkeit von sprachlichen Konstruktionen. Wenn mehrere Informanten an einer Feststellung der Richtigkeit einer Aussage beteiligt sind, so können die Ergebnisse einer solchen 
Untersuchung zur Formulierung eines „durchschnittlichen Sprachgefühls“ (vgl. Juhász 1970, S. 42) verhelfen.

Das Sprachgefühl spielt auch eine wichtige Rolle bei der Interferenz-Frage. Es ist als erste Beurteilungsinstanz bei der Suche bzw. beim Erkennen sprachlicher (Interferenz) Fehler anzusehen. Bei der Rezeption eines sprachlichen Ausdrucks gibt uns das Sprachgefühl ein Signal, dass der Ausdruck nicht korrekt ist. Erst dann kommt es zu einer bewussten Überlegung und Konfrontierung des Ausdrucks mit den im Kopf des Rezipienten angesammelten Normen und Regeln. Das Sprachgefühl vermag die oben genannten Normen nicht völlig zu ersetzen. Es ist kein festes Regelwerk und kann in manchen Fällen täuschend sowie irreführend sein. Daher soll das Sprachgefühl nicht als einzige bzw. letzte Beurteilungsinstanz angesehen werden.

Beim Sprachgefühl geht es darum, die sprachlichen Aussagen so zu beurteilen, dass der Sprachbenutzer aus den gegebenen Ausdrucksmöglichkeiten die richtige wählt. Um eine aufschlussreiche Sprachanalyse mit Hilfe des Sprachgefühls durchführen zu können, ist es nötig, eine entsprechende Menge von Informanten zu befragen. Juhász weist darauf hin, dass bei einer solchen Analyse das Sprachgefühl von Versuchsperson zu Versuchsperson bzw. von Situation zu Situation variiert. Auch solche Faktoren wie Intelligenz, Ausbildung, (sprachliche) Gewohnheiten, psychischer und physischer Zustand, schriftliche oder mündliche Ausdrucksweise oder die Person des Gesprächspartners haben einen nicht zu unterschätzenden Einfluss auf das Sprachgefühl (vgl. Juhász 1970, S. 44). Diese Differenzen und Streuungen bei den Informanten sind insofern wichtig, als sie es unmöglich machen, aus den Befragungsergebnissen eine Norm zu bestimmen. Man kann sich auf die Ergebnisse nicht immer verlassen, weil die Faktoren, die das Sprachgefühl determinieren und bestimmen, uneinheitlich sind. Nichtdestotrotz schreibt Károly zum Sprachgefühl: „(...) es ist der wichtigste Sprachregler; ohne sich darauf zu stützen, ist keine wirkliche Sprachbeherrschung möglich" (Károly 1966, S. 333). Juhász bemerkt, dass das Sprachgefühl zum Sprechen und zur Beurteilung sprachlicher Ausdrücke notwendig ist. Des Weiteren führt er aus, dass jedes individuelle Sprachgefühl langue auf seine eigene Weise realisiert (vgl. Juhász 1970, S. 45), so dass sich die Urteile zur Korrektheit einer gegebenen Aussage erheblich voneinander unterscheiden können. Dies bezieht sich allerdings nur auf die Zweifelsfälle, die einen relativ kleinen Teil der einzuschätzenden Aussagen betreffen (die entscheidende Mehrheit der Fälle ist unproblematisch).

Juhász macht auch eine aufschlussreiche Synthese verschiedener Versuche zur Definition von dem, was man unter „Sprachgefühl“ versteht. Hierbei ergeben sich folgende Auffassungen des Phänomens:

- ein dunkles, unterschwellig wirksames Wissen um das Sprachübliche, eine durch weitgehende Sprachvertrautheit geschaffene und ständig verfeinerte Disposition, sich der Bestände der betreffenden Sprache in normgemäßer Weise zu bedienen. 
- ein automatisierter und betriebsförmig gewordener (funktionalisierter) Kenntnisbesitz in Form eines gefügigen Bereithabens von Leitschematen und Modellkonstruktionen, die als zuhandener Übungserwerb ohne diskursive Besinnungsakte zur Verfügung stehen.

- ein verdichteter Erfahrungsniederschlag, der als Resultante von Gedächtnis und Analogie ein konkretes Operieren mit den Beständen der Sprache sowie ein zutreffendes Bewerten sprachlicher Fügungen ermöglicht, und zwar ohne volle Bewußtseinserhellung, ferner ohne Vorhandensein und Präsenz expliziter theoretischer grammatisch-stilistischer Kenntnisse, also ohne logische Klarheit der Entscheidungsgründe.

- Im Sprachgefühl vereinigen sich rezeptive und produktive, ferner impressive und aktiv-expressive Faktoren; es gestattet ein Erkennen und Verbessern fehlerhafter Sprachfügungen anderer, sodann ermöglicht es uns das korrekte Spontansprechen und-schreiben, d.h. die Angleichung des von uns selbst Produzierten an den als Norm geltenden Sprachgebrauch. Es ist in der Regel das Sprachgefühl, das die Übereinstimmung der Redeleistung (,parole`) als einer individuellen Handlung mit dem konventionellen Regelsystem einer bestimmten ,langue' herstellt (...) (Juhász 1970, S. 43f.).

Aus der oben genannten Synthese kann man folgende Schlussfolgerungen ziehen: 1. Sprachbenutzer sind sich dessen nicht bewusst, dass sie einen Ausdruck mit Hilfe ihres Sprachgefühls prüfen.

2. Der Vorgang der Feststellung von Korrektheit einer Aussage geht automatisch vor sich; man braucht hierbei nicht zusätzlich zu handeln.

3. Das Sprachgefühl macht zutreffendes Bewerten der sprachlichen Aussagen möglich.

4. Es sind keine theoretischen Kenntnisse nötig, damit aus dem Sprachgefühl Gebrauch gemacht werden kann.

5. Bei dem Phänomen ,Sprachgefühl“ spielen mehrere Faktoren mit, die rezeptiver, produktiver, impressiver und aktiv-expressiver Natur sein können.

Conrad gebraucht für das Sprachgefühl auch die Bezeichnung „Intuition“ und versteht es folgendermaßen:

Intuition od. Sprachgefühl: Gesamtheit der erworbenen Kenntnisse (Regeln) von der Muttersprache oder einer Fremdsprache, die unbewußt beim aktiven Sprachgebrauch angewendet werden. Beruht auf der angeborenen menschlichen Fähigkeit, Sprachen zu lernen und sich somit solche Regeln anzueignen. I. wird durch die Grammatik bewußt gemacht und in einen erklärenden Zusammenhang gebracht (Conrad 1988, S. 105).

Damit weist er darauf hin, dass die Menschen von Geburt an die Sprache lernen können. Diese Fähigkeit ermöglicht ihnen, sich ein Regelwerk anzueignen, das als „Sprachgefühl“ bezeichnet wird. Das Wort „Intuition“ impliziert für Conrad einen unbewussten Vorgang der Speicherung und Anwendung der Regeln. Die Grammatik macht die Intuition bewusst, was allerdings nichts an der Tatsache ändert, dass die Bewusstmachung etwas schon früher Existierendes betrifft. Das Sprachgefühl gewinnt dann eine Stütze in Form von grammatischen Regeln. Die Regeln sind nicht notwendig - in vielen Fällen kann ein Sprachbenutzer einen sprachlichen Ausdruck nur mit Hilfe des Sprachgefühls beurteilen. Natürlich kann es zu 
einer Situation kommen, wo es Zweifel gibt, ob eine Form (z.B. bei der Interferenz-Untersuchung) als fehlerhaft oder - frei einzustufen ist. Gaworski formuliert es in folgenden Worten:

In Zweifelsfällen muss sich deshalb der Untersuchende auf sein Sprachgefühl verlassen, um zu entscheiden, ob man eine nicht regelkonforme muttersprachliche Äußerung als potentielle Interferenzquelle in die Analyse einbeziehen soll (Gaworski 2007, S. 38).

Das Sprachgefühl kann sich auch auf die Fremdsprache beziehen. Der Hauptunterschied zwischen dem Sprachgefühl für die Muttersprache und für die Fremdsprache liegt in der Automatisierung der sprachlichen Strukturen. Im Fall der Muttersprache sind die Strukturen schon automatisiert, wogegen sie bei der Fremdsprache erst automatisiert werden müssen. Dies hängt vom Niveau des Fremdsprachenlernenden ab. Auf der Anfangs- bzw. Mittelstufe kann verständlicherweise kaum von einem Sprachgefühl für die Fremdsprache gesprochen werden. Die Fähigkeiten, die bei dem Sprachgefühl mitspielen und die Beurteilung sprachlicher Aussagen ermöglichen, müssen erst erworben werden, was schrittweise erfolgt. Dabei kommen zu viele Faktoren und Determinanten in Betracht, als dass es möglich wäre, sie auf einmal bzw. schnell zu automatisieren. Die Zahl der automatisierten Strukturen wird mit der Zeit größer. Hierbei muss man bemerken, dass schon auf der Mittelstufe gewisse Strukturen automatisiert sein können. Für Juhász ist jedoch ihre Zahl im Vergleich mit dem ganzen Korpus der Sprache so klein, dass man in dem Fall von keinem Sprachgefühl sprechen kann (vgl. Juhász 1970, S. 45). Dafür spricht auch die Tatsache, dass die einzelnen zu erwerbenden Sprachfertigkeiten eng miteinander verflochten sind.

\section{LITERATURVERZEICHNIS}

Althaus H., Henne H., Wiegand H. (Hrsg.) (1980), Lexikon der Germanistischen Linguistik, Tübingen.

Bondzio W. (1980), Einführung in die Grundfragen der Sprachwissenschaft, Leipzig.

Conrad R. (1988) (Hrsg.), Lexikon sprachwissenschafticher Termini, Leipzig.

Duden, Deutsches Universalwörterbuch (2003), Mannheim.

Gaworski I. (2007), Auswirkungen interlingualer Interferenz auf die deutsche Wort- und Satzgliedstellung im schriftlichen Bereich, Wrocław-Dresden.

Glück H. (Hrsg.) (1993), Metzler Lexikon Sprache, Metzler, Stuttgart/Weimar.

Juhàsz J. (1970), Probleme der Interferenz, München.

Károly S. (1966), Mejegyzések 'A jelentés kérdései ‘-hez (Antal Lászlo tanulmányához). In: Nyelvtudományi Közlemények 68, S. 327-342.

Markowski A. (Hrsg.) (2005), Wielki słownik poprawnej polszczyzny PWN, Warszawa.

Nerius D. (1967), Untersuchungen zur Herausbildung einer nationalen Norm der deutschen Literatursprache im 18. Jahrhundert, Halle.

Semenjuk N. (1975), Die sprachliche Norm. In: Serebrennikow B. (Hrsg.), Allgemeine Sprachwissenschaft, Bd. 1, Berlin, S. 454- 494.

Skudrzykowa A., Urban K. (2000), Mały stownik terminów z zakresu socjolingwistyki i pragmatyki językowej, Kraków/Warszawa. 
Tomasz Maras

\section{THE LINGUISTIC NORM AND ITS (IN)VARIANTS AS A BASIS FOR THE EVALUATION OF LINGUAL EXPRESSIONS}

(Summary)

The main subject of this article is the linguistic norm and its (in)variants with regard to evaluation of lingual expressions. The article tries to show various German and Polish conceptions of linguistic norms and to point to their reference to lingual errors/mistakes and interference. Functional norm, usus, feel for language and their importance for language standardization are also discussed in the article, together with the relativity of linguistic norm.

Key words: linguistic norm, lingual errors/mistakes, interference, usus, language standardization. 